\title{
Maternal Deprivation Induces Memory Deficits That Are Reduced by One Aerobic Exercise Shot Performed after the Learning Session
}

\author{
Priscila Marques Sosa, ${ }^{1}$ Ben-Hur S. Neves, ${ }^{1}$ Guilherme Salgado Carrazoni, ${ }^{1}$ \\ Gabriela Mendes Gomes, ${ }^{1}$ Gabriel Del Rosso, ${ }^{1}$ Bruna Piaia Ramborger, ${ }^{2}$ Rafael Rohers, ${ }^{2}$ \\ and Pâmela Billig Mello-Carpes $\mathbb{D}^{1}$
}

${ }^{1}$ Physiology Research Group, Stress, Memory and Behavior Lab, Federal University of Pampa, Uruguaiana, RS, Brazil

${ }^{2}$ Grupo Interdisciplinar de Pesquisa em Prática de Ensino, Universidade Federal do Pampa, Uruguaiana, RS, Brazil

Correspondence should be addressed to Pâmela Billig Mello-Carpes; panmello@hotmail.com

Received 2 August 2019; Revised 16 October 2019; Accepted 29 October 2019; Published 16 November 2019

Academic Editor: Gabriela Delevati Colpo

Copyright (C) 2019 Priscila Marques Sosa et al. This is an open access article distributed under the Creative Commons Attribution License, which permits unrestricted use, distribution, and reproduction in any medium, provided the original work is properly cited.

\begin{abstract}
During the neonatal period, the brain is susceptible to external influences. Exposure to stressful events during this phase of life influences brain development and impacts adult life. In animals, the maternal deprivation (MD) model is effective in mimicking stress in the early stages of development. In contrast, physical exercise seems to be able to prevent deficits in memory consolidation. Although the effects of chronic exercise in cognition are already well established, little is known about the effects of acute aerobic exercise. Here, male Wistar rats divided into deprived (MD) and nondeprived (NMD) rats were submitted to the object recognition (OR) memory test. Immediately after OR training, some of the rats were submitted to a single aerobic exercise session for 30 minutes. Memory consolidation and persistence were evaluated by retention tests performed $24 \mathrm{~h}$ and 7 , 14 , and 21 days after OR training. We show that a single physical exercise session is able to modulate learning by promoting memory consolidation and persistence in rats with cognitive deficits induced by MD. Hippocampal dopamine levels, measured by HPLC, were not altered after OR training in rats that performed and in rats that did not perform an exercise session; on the other hand, while OR training promoted increase of hippocampal norepinephrine in NMD rats, the MD rats did not present this increase, regardless of the practice or not of exercise.
\end{abstract}

\section{Introduction}

Exposure to stressful environmental events during early childhood may impact neural plasticity and increase the vulnerability to psychopathologies [1]. The early life stress causes neuroendocrine changes associated with social and emotional behavior alterations [2] that may persist throughout life [3]. In humans, especially from the prenatal period until the early years of postnatal life, the brain undergoes a rapid development [4] and is highly sensitive to the influence of positive and negative external experiences [5].

An example of early childhood stress is the maternal separation; in humans, it can be related to institutionalization in orphanages [6], which has already been shown to be responsible for long-term behavioral damages, such as increased anxiety [7], and psychopathologies, as schizophrenia [8]. Several reports suggest that early childhood traumas may affect distinct neural circuits, such as those involving the dopaminergic and the noradrenergic systems [9-13], which perform several functions in the brain, including the regulation of cognition, attention, and stress [12, 14-17]. In addition, neuroimaging studies in humans suggest that early childhood stress interferes in the maturation of the dopaminergic system [18].

In rodents, maternal deprivation (MD) is a stress model widely used as a paradigm to study early life adverse events [19], as parental abuse or loss [11]. In this model, there is evidence that early-stage stress leads to changes in dopamine 
neurotransmission, affecting both dopamine (DA) levels [20] and dopaminergic receptor density (D1 and D2) [18]. In addition to interactions with the dopaminergic system, evidences suggest that early childhood stress also changes noradrenergic neural networks such as the locus-coeruleusnorepinephrine (NE) system $[21,22]$.

In contrast, some strategies have been used to improve brain DA and/or NE levels, as well as cognitive functions. One is the physical exercise. Chronic aerobic exercise effects on cognition are widely studied $[23,24]$, and researches demonstrated that exercise improves behavioral, mental health $[25,26]$ and biochemical parameters $[23,27,28]$. Despite other mechanisms involved on the aerobic exercise effects, it acts by exciting the central nervous system through positive regulation of catecholamines, including epinephrine (E), NE, and DA [29-32]. Furthermore, a previous study from our laboratory demonstrated that a single exercise session is able to promote object recognition memory persistence, which is related to the increase of NE levels [33].

Thus, in the present work, we aim to investigate the effects of a single aerobic exercise session on memory deficits related to $\mathrm{MD}$ in rats. Additionally, we investigated the impact of MD and a single aerobic exercise session on hippocampal DA and NE levels.

\section{Materials and Methods}

2.1. Animals and Experimental Design. Pregnant female Wistar rats were obtained from the Central Vivarium of the Federal University of Santa Maria (RS/Brazil). All animals were kept in a light/dark cycle of 12 h (7:00 a.m. to 7:00 p.m.) at controlled ambient temperature $\left(23 \pm 2^{\circ} \mathrm{C}\right)$ and air humidity $(60 \pm 5 \%)$. The rats were housed individually, with food and water ad libitum.

The delivery day was considered as day zero. From the postnatal day 1 (PND-1) to the PDN-10, the MD protocol was performed with half of the pups, as described below. The animals were weaned in PND-21 and housed 5 per cage. Only males were used in the experiments described in this paper. All experiments were performed according to the principles of laboratory animal care (NIH Publication No. 80-23, revised 1996) and approved by the Institutional Committee on Animal Care and Use of the Federal University of Pampa (RS/Brazil) (050/2017).

The rats were divided into six groups, according the procedures adopted, which are described below.

(i) NMD. Control rats were not submitted to the MD protocol but were trained in the object recognition memory (OR) task $(n=15)$

(ii) $M D$. The rats were submitted to the MD protocol and were trained in the OR task $(n=15)$

(iii) $N M D+F A E$. The rats were not submitted to the MD protocol, were familiarized to the exercise apparatus (a treadmill for rodents), and were trained in the OR task, without the exercise shot after OR training $(n=15)$ (iv) $M D+F A E$. The rats were submitted to the MD protocol, were familiarized to the exercise apparatus, and were trained in the OR task, without the exercise shot after OR training $(n=15)$

(v) $N M D+A E$. The rats were not submitted to the MD protocol, were familiarized to the exercise apparatus, were trained in the OR task, and were submitted to an aerobic physical exercise shot immediately after $(n=15)$

(vi) $M D+A E$. The rats were submitted to the MD protocol, were familiarized to the exercise apparatus, were trained in the OR, and were submitted to an aerobic physical exercise shot immediately after OR training $(n=15)$

After, ten rats from each group were submitted to $\mathrm{OR}$ memory testing $24 \mathrm{~h}$ and 7, 14, and 21 days after OR training (Figure 1). In all testing days, the Elevated Plus Maze task was performed after OR testing, as an anxiety control test. Five animals from each group were euthanized immediately after the exercise shot, their brains were isolated, and the hippocampi were quickly dissected for use in biochemical tests. For biochemical tests, additional naïve rats were used $(n=4)$; these animals were not submitted to any behavioral procedure.

2.2. Maternal Deprivation. Female Wistar rats were kept in individual boxes until the delivery day (day 0 ). The rats of groups ii, iv, and vi were submitted to maternal deprivation (MD) for 10 days (PND-1 to PND-10), 3 hours/day, always during the light part of the light/dark cycle.

The MD protocol consisted in removing the mother from the residence box to another room, without touching the puppies. Pups were kept in their cage, and while the mothers were absent, the room temperature was maintained at around $32^{\circ} \mathrm{C}$ to compensate the loss of the mother's body heat [34]. At the end of each daily deprivation session, the mothers were returned to their original boxes.

The rats of groups $\mathrm{i}$, iii, and $\mathrm{v}$ remained in their residence boxes with their mothers until PND-21. On PND-21, all rats were weaned, and males were kept in groups of 5 in plastic boxes, similar to all other animals in our accommodation facility. The female pups were donned to other experiments. This specific MD protocol was selected since in previous studies no changes in anxiety or other behavioral parameters that could interfere with the memory testing were found [34-36].

2.3. Familiarization with the Exercise Apparatus. Prior to the memory task, rats were familiarized with the treadmill where the physical exercise would be performed to avoid novelty and/or stress effects. Initially, the rats were familiarized with the treadmill for three days (first day static and then with treadmill at a speed of 2 to $5 \mathrm{~m} / \mathrm{min}$ for $10 \mathrm{~min}$ ).

Afterwards, they were submitted to the "good runner protocol," which consists in placing the animals on a treadmill without slope for three consecutive days (velocity of $8 \mathrm{~m} / \mathrm{min}$ for $10 \mathrm{~min}$ ) and then assessing the level of 


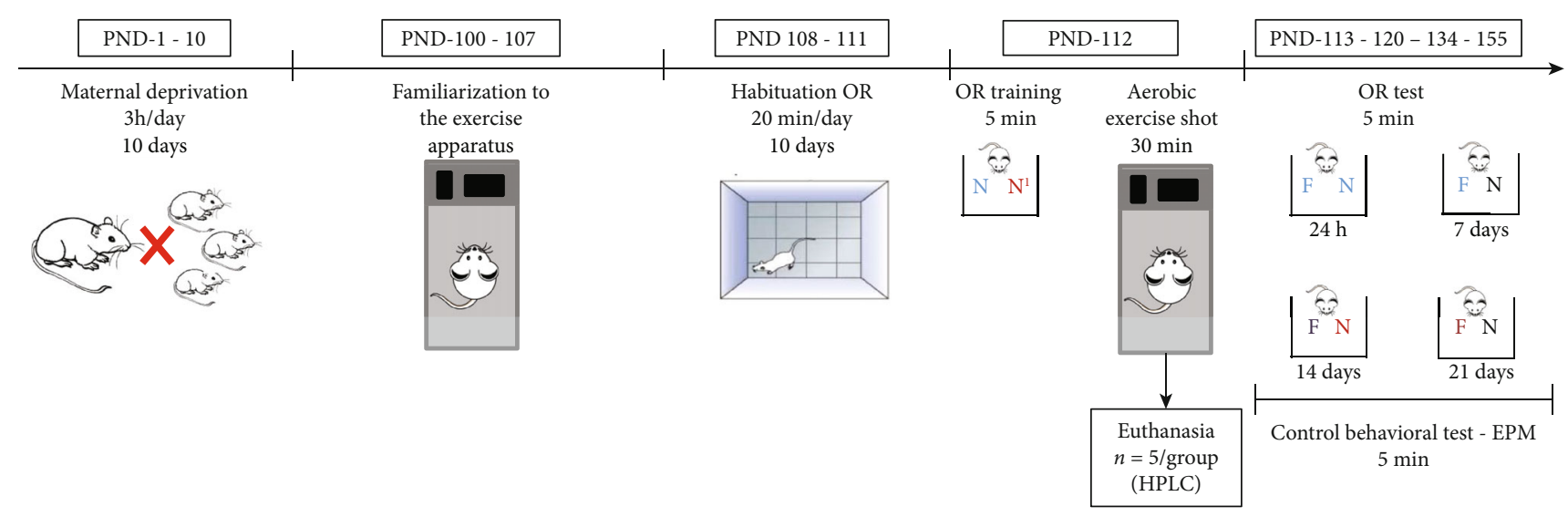

FIgURE 1: Experimental design. Half of the animals were submitted to the maternal deprivation (MD) protocol from PND-1 to PND-10 (groups ii, iv, and vi). Animals from groups iii to vi were familiarized to the physical exercise apparatus from PND 100 to PND 107. All animals, except the naïve, were habituated to the object recognition (OR) memory task apparatus (PND 108-111) and trained in the OR task (PND112), when they were exposed to two different new $(\mathrm{N})$ objects for free exploration. Animals of groups v and vi were submitted to an aerobic exercise shot immediately after OR training. After the aerobic exercise shot, 5 animals from each group, plus 4 naïve animals, were euthanized for biochemical analysis (HPLC). The other animals were submitted to OR tests $24 \mathrm{~h}$ and 7,14 , and 21 days after the OR training (PNDs 113, 120,134, and 155), when the animals were exposed to a familiar (F) and to a new object (N) for free exploration. On each testing day, always after the OR test session, the Elevated Plus Maze (EPM) test was performed as a control behavioral test to verify anxiety.

trainability, considering a scale from 1 to 5 points (1: refuses to run; 2: runners below average (runs and stops or runs in the wrong direction); 3: average runner; and 4: above average (runs well), with sporadic stops on the treadmill); in the end, rats that maintained a mean of three or more points were included in the exercise groups [37].

On the last day, an indirect $\mathrm{VO}_{2}$ maximum test was performed to determine individual exercise intensity (starting at a slow rate increased by $5 \mathrm{~m} / \mathrm{min}$ every 3 minutes until the rat could not continue to run) $[38,39]$.

\subsection{Habituation and Training in the Object Recognition} Memory Task. The object recognition (OR) training and tests were performed in an arena $(50 \times 50 \times 50 \mathrm{~cm})$ constructed of polyvinyl chloride, plywood, and transparent acrylic, as described by Ennaceur and Delacour [40]. The sessions were filmed for further analyses, and the experimenters were blinded to animals' experimental condition. Firstly, the animals were familiarized to the OR apparatus by placing them inside the arena for $20 \mathrm{~min}$ per day to freely explore on 4 consecutive days prior to OR training. On the training day, two different objects $\left(\mathrm{N}\right.$ and $\left.\mathrm{N}^{1}\right)$, equally attractive for the rats, were placed in the apparatus and the animals were free to explore them for $5 \mathrm{~min}$. The objects were made of metal, glass, or glazed pottery. The time exploring each object was accounted.

2.5. Aerobic Exercise Shot. Animals from groups v and vi were submitted to a single physical exercise session immediately after the OR training, using a treadmill built for rodents (Insight Ltda., São Paulo, Brazil). The protocol used was adapted from Malek et al. [41]; an intensity of $60-70 \%$ of maximum oxygen consumption $\left(\mathrm{VO}_{2}\right)$ in a single session for $30 \mathrm{~min}$ was used.
2.6. Object Recognition Memory Tests. Twenty-four hours and 7,14 , and 21 days after training, in the memory testing phase, one of the objects used in OR training was maintained (Familiar object-F) and the other was randomly replaced by a new one $(\mathrm{N})$. In each test day, the objects were placed in the apparatus and the animals were free to explore them for $5 \mathrm{~min}$. The test sessions were recorded, and after, the time exploring each object was accounted.

2.7. Elevated Plus Maze (EPM) Test. To assess the anxiety state, which could affect the results of memory tests, the rats were exposed to EPM in each testing days, always after the OR test. The EPM apparatus consists of a raised platform, located $60 \mathrm{~cm}$ above the ground, and has two closed arms face to face and two open arms, each measuring $50 \times 10 \mathrm{~cm}$, containing infrared sensors. The closed arms are composed of sidewalls $20 \mathrm{~cm}$ high. The time spent and the total number of entries in the open and closed arms were recorded in a 5-minute session [42].

2.8. Biochemical Testing. Five rats from groups i to vi were sacrificed immediately after the single session of aerobic exercise or in the equivalent time. Additionally, 4 naïve groups were sacrificed. The rats' brains were quickly removed for dissection of the bilateral hippocampus. The tissues were homogenized in $50 \mathrm{mM} \mathrm{HCl}, \mathrm{pH} 7.4(1 / 5, w / v)$. Subsequently, the samples were centrifuged at $2400 \mathrm{~g}$ for $10 \mathrm{~min}$, and the supernatants (S1) were filtered and then stored at $-80^{\circ} \mathrm{C}$ for further analysis.

The levels of DA and NE in hippocampus homogenates were determined using an "Inertsil ODS-3" reverse-phase High Performance Liquid Chromatography (HPLC) system $(5 \mu 4.6 \times 250 \mathrm{~mm}$, GL Sciences) with a diode array detector as described by [43]. The mobile phase consisted of methanol and water $(12 / 88, v / v)$ adjusted to $\mathrm{pH} 3$ with phosphoric acid. 
The concentrations of the neurotransmitters relative to the standard analytical curve equation were obtained and corrected according to the dilution and sample weight.

To separate DA and NE, we used the isocratic programming with a flow rate of $0.8 \mathrm{~mL} / \mathrm{min}$. The sample was filtered through $0.22 \mu \mathrm{m}$ syringe filters. We inject $20 \mu \mathrm{L}$ of samples into the HPLC system by an automatic sampling device (YL9150). Detection was at $198 \mathrm{~nm}$ by DAD. Chromatograms were recorded and integrated by PC integration software (YLClarity). All analyses were performed in triplicate. Analytical parameters were as follows: linear range, 0.1$10.0 \mu \mathrm{g} / \mathrm{mL}$; coefficient of determination, 0.999; and calibration equation, $y=628,12 x-34,342$. DA and NE for HPLC were supplied by Sigma-Aldrich, Brazil. Other reagents used in this experiment were from analytical grades and obtained from standard commercial suppliers.

2.9. Statistical Analyses. The data distribution was analyzed using the Shapiro-Wilk test.

The exploration time of the objects in the OR task was converted to a percentage of the total exploration time on the training or test sessions, and the Wilcoxon signed-rank test was used to compare the percentage of the total exploration time spent in each object considering a theoretical mean of $50 \%$.

The EPM data were compared by the Kruskal-Wallis test.

The HPLC results and total exploration time in each OR session were compared by ANOVA, followed by Tukey's multiple comparison tests.

The results are expressed as the mean $\pm \mathrm{SD}$. In all analyses, $P$ values $<0.05$ were considered significant.

\section{Results}

3.1. Object Recognition Memory Consolidation and Persistence. Memory consolidation and persistence were assessed by the OR test. During OR training, the rats from all groups explored the two objects by a similar percentage of total exploration time, $\sim 50 \%$ of the total exploration time each one $\left(P=0.704, t_{(8)}=0.393\right.$ for NMD group; $P=0.242, t_{(9)}=1.288$ for $\mathrm{MD}$ group; $P=0.155$, $t_{(9)}=1.552$ for NMD+FAE group; $P=0.190, t_{(9)}=1.416$ for $\mathrm{MD}+\mathrm{FAE}$ group; $P=0.460, t_{(7)}=0.780$ for $\mathrm{NMD}+\mathrm{AE}$ group; and $P=0.310, t_{(9)}=1.075$ for $\mathrm{MD}+\mathrm{AE}$ group; Figure 2(b)).

In the $24 \mathrm{~h}$ test, the control group (NMD) explored significantly more than $50 \%$ of the new object $(P=0.039$, $t_{(8)}=2.513$; Figure $\left.2(\mathrm{c})\right)$. In persistence tests, this difference was not observed (for 7: $P=0.250, t_{(8)}=1.718$, Figure $2(\mathrm{~d}$ ), NMD; for 14: $P=0.062, t_{(6)}=0.038$ exploring the familiar object in relation to the new object, Figure 2(e), NMD; and for 21 days: $P=0.734, t_{(9)}=0.168$, Figure 2(f), NMD).

As expected, MD rats did not consolidate memory. On the $24 \mathrm{~h}$ test, they explored $\sim 50 \%$ of the total exploration time each object $\left(P=0.570, t_{(9)}=0.8847\right.$; Figure $\left.2(\mathrm{c}), \mathrm{MD}\right)$, which is observed until the 21-day test $\left(P=0.125, t_{(7)}=1.84\right.$ for 7 -day test, Figure 2(d), MD; $P=0.082, t_{(9)}=1.972$ for 14-day test, Figure 2(e), MD; and $P=0.406, t_{(9)}=0.916$ for 21-day test, Figure 2(f), MD).

Nondeprived rats (NMD) familiarized to the exercise apparatus (NMD+FAE) explored more than $\sim 50 \%$ of the new object in comparison to the familiar one in the $24 \mathrm{~h}$ test $\left(P=0.002, t_{(9)}=4.239\right.$, Figure 2(c), NMD+FAE). The memory persisted up to 14 days after the training $\left(P=0.002, t_{(9)}=6.663\right.$ for 7 -day test, Figure 2(d), NMD+ FAE; $P=0.037, t_{(9)}=2.868$ for 14 -day test, Figure 2(e), $\mathrm{NMD}+\mathrm{FAE})$. Memory persistence was not observed on the 21 -day test $\left(P=0.064, t_{(9)}=2.245\right.$, Figure 2(f), NMD+FAE).

The MD rats familiarized to the exercise apparatus (MD+ FAE) explored significantly more than $50 \%$ of the new object in the $24 \mathrm{~h}$ test $\left(P=0.019, t_{(9)}=3.402\right.$; Figure $2(\mathrm{c}), \mathrm{MD}+$ FAE) but not in the memory persistence tests $(P=0.05$, $t_{(9)}=2.255$ for the 7-day test, Figure $2(\mathrm{~d}), \mathrm{MD}+\mathrm{FAE} ; P=$ $0.160, t_{(9)}=1.724$ for the 14-day test, Figure 2(e), MD+ FAE; and $P=0.652, t_{(9)}=0.507$ for the 21-day test, Figure 2(f), MD+FAE).

Nondeprived rats who performed aerobic exercise on the treadmill (NMD+AE) explored significantly more than $\sim 50 \%$ of the new object in the $24 \mathrm{~h}$ test $\left(P=0.015, t_{(7)}=\right.$ 3.429, Figure 2(c), NMD+AE). Memory persistence was observed until 14 days after training $\left(P=0.015, t_{(7)}=3.617\right.$ for 7-day test, Figure 2(d), NMD+AE; $P=0.031, t_{(7)}=$ 2.784 for 14-day test, Figure 2(d), NMD+AE). On the test performed 21 days after training, memory persistence was not observed $\left(P=0.312, t_{(7)}=1.215\right.$, Figure $\left.2(f), N M D+A E\right)$.

The maternal-deprived rats submitted to physical exercise shot $(\mathrm{MD}+\mathrm{AE})$ presented a good memory consolidation, since they explored the new object for more than $50 \%$ of the total exploration time in the $24 \mathrm{~h}$ test $\left(P=0.002, t_{(9)}=4.774\right.$; Figure $2(\mathrm{c}), \mathrm{MD}+\mathrm{AE})$. In addition, the memory persisted for 21 days $\left(P=0.007, t_{(8)}=4.429\right.$ for 7 -day test, Figure $2(\mathrm{~d})$, $\mathrm{MD}+\mathrm{AE} ; P=0.003, t_{(9)}=4.140$ for 14 -day test, Figure 2(e), $\mathrm{MD}+\mathrm{AE}$; and $P=0.003, t_{(9)}=4.057$ for 21 -day test, Figure 2(f), $\mathrm{MD}+\mathrm{AE}$ ).

It is importantly highlighted that despite the differences between groups in the percent of time spent exploring the new and the familiar objects in some testing sessions, no differences in the total exploration time were found between the groups in any OR session (Table 1).

3.2. Elevated Plus Maze. MD and aerobic physical exercise protocol did not alter the anxiety of the animals in the testing session performed $24 \mathrm{~h}$ and 7,14 , and 21 days after training (Table 2).

3.3. Catecholamine Levels. No differences were found between groups on hippocampal dopamine levels $\left(F_{(6,95)}=1.33 ; P=0.2513\right.$, Figure $\left.3(\mathrm{a})\right)$. On the other hand, differences were found on hippocampal norepinephrine levels between groups $\left(F_{(6,54)}=13.08 ; \quad P<0.0001\right.$, Figure 3(b)): NMD groups presented higher NE levels than naïve animals $(P<0.001)$. MD groups presented lower $\mathrm{NE}$ levels than NMD groups $(P<0.05$ for all comparisons, 
Exercise and OR protocol summary

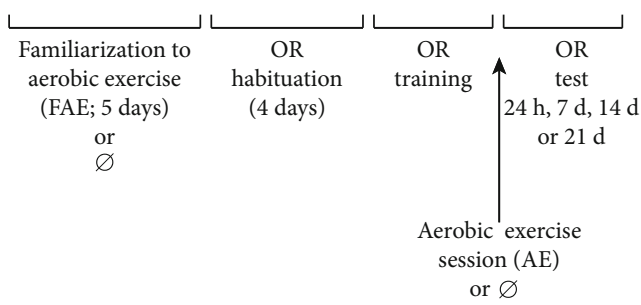

(a)

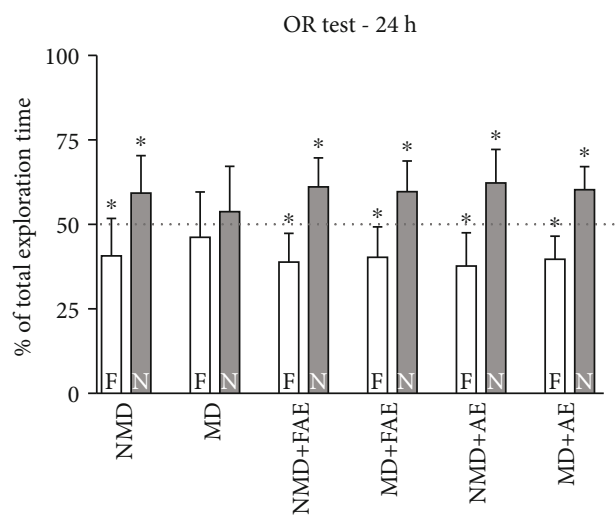

(c)

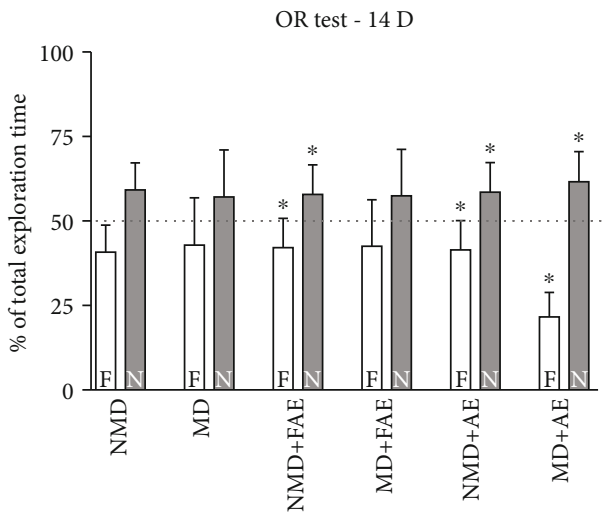

(e)

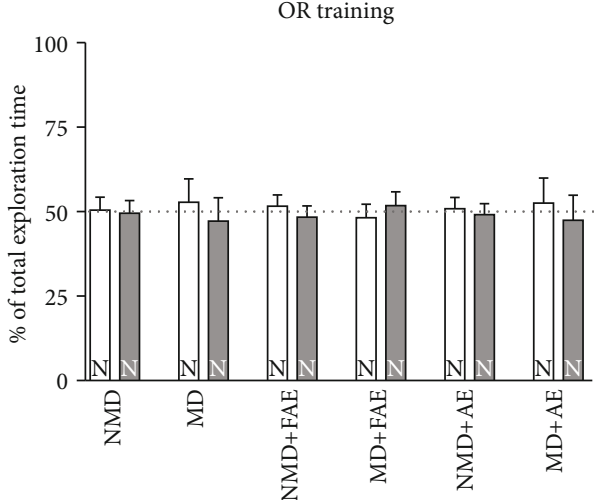

(b)

OR test - $7 \mathrm{D}$

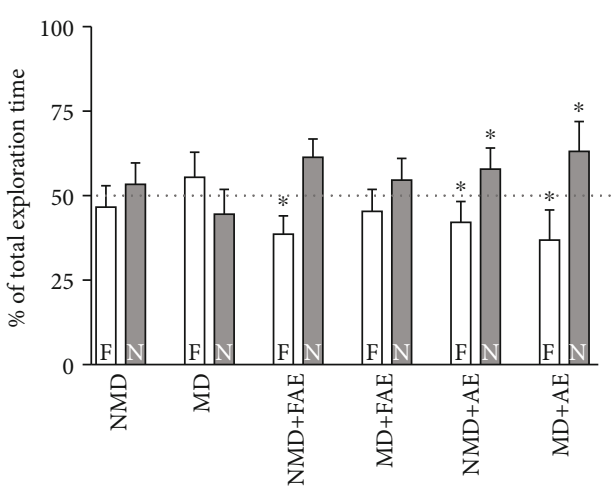

(d)

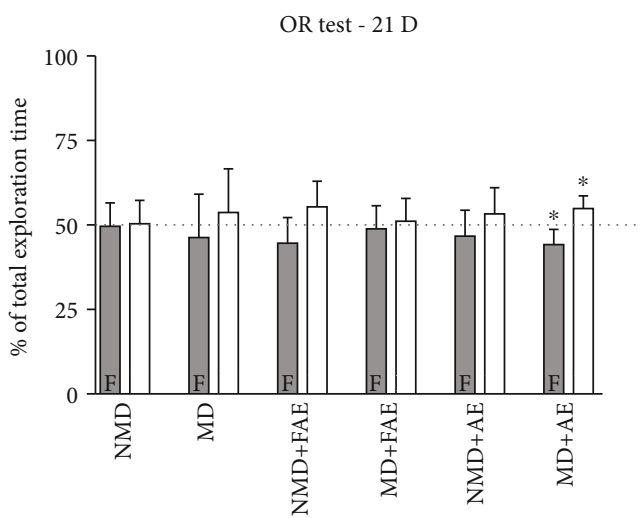

(f)

Figure 2: Maternal deprivation (MD) causes long-term memory deficits of object recognition (OR) memory. An acute aerobic exercise session is able to reverse these deficits. (a) The animals were trained in the OR task and tested 24 hours and 7, 14, and 21 days after; animals from some groups were submitted to familiarization of aerobic exercise in a treadmill (NMD+FAE, MD+FAE, NMD+AE, and $\mathrm{MD}+\mathrm{AE}$ ), and animals from some groups were submitted to an aerobic exercise session just after the OR training session (NMD+AE and $\mathrm{MD}+\mathrm{AE})$. (b) In the training session, the animals were exposed to two new objects (N), and animals from all groups explored about $50 \%$ of the total exploration time each one. (c) In the OR consolidation memory test $(24 \mathrm{~h})$, the rats were exposed to a familiar (F) and to a new object $(\mathrm{N})$. The MD group was not able to distinguish the familiar from the new object in the test session but the MD rats familiarized to treadmill (FAE) and submitted to a shot of physical exercise (AE) were. When tested 7 (d) and 14 (e) days after training, neither the MD group nor the NMD group was able to distinguish the familiar and new objects. All rats that performed acute physical exercise were able to distinguish the familiar object from the new one. (f) After 21 days, only MD rats submitted to exercise present persistence of memory. The data are expressed as the mean \pm standard deviation of the percentage of total exploration time; ${ }^{*} P<0.05$ for the Wilcoxon signed-rank test, considering a theoretical mean of $50 \%$; $n=8-10$ per group. $\mathrm{OR}=$ object recognition test; $\mathrm{NMD}=$ nonmaternal-deprived rats; $\mathrm{MD}=$ maternal-deprived rats; $\mathrm{NMD}+\mathrm{FAE}=$ nonmaternal-deprived rats submitted to aerobic exercise familiarization; $\mathrm{MD}+\mathrm{FAE}=$ maternal-deprived rats submitted to aerobic exercise familiarization; $\mathrm{NMD}+\mathrm{AE}=$ nonmaternal-deprived rats submitted to aerobic exercise familiarization and to an aerobic exercise session; $\mathrm{MD}+\mathrm{AE}=\mathrm{maternal}-$ deprived rats submitted to aerobic exercise familiarization and to an aerobic exercise session. 
TABLE 1: Total exploration time in training and tests on the OR task. Data are expressed as the mean \pm SD of the total exploration time, in seconds, on OR training and tests $(\mathrm{OR} ; n=8-10$ per group/day; $P>0.05$; ANOVA). NMD = nonmaternal-deprived rats; $M D=$ maternaldeprived rats; $\mathrm{NMD}+\mathrm{FAE}=$ nonmaternal-deprived rats submitted to aerobic exercise familiarization; $\mathrm{MD}+\mathrm{FAE}=$ maternal-deprived rats submitted to aerobic exercise familiarization; $\mathrm{NMD}+\mathrm{AE}=$ nonmaternal-deprived rats submitted to aerobic exercise familiarization and to an aerobic exercise session; $\mathrm{MD}+\mathrm{AE}=$ maternal-deprived rats submitted to aerobic exercise familiarization and to an aerobic exercise session.

\begin{tabular}{|c|c|c|c|c|c|c|c|}
\hline & NMD & $\mathrm{MD}$ & $\mathrm{NMD}+\mathrm{FAE}$ & $\mathrm{MD}+\mathrm{FAE}$ & $\mathrm{NMD}+\mathrm{AE}$ & $\mathrm{MD}+\mathrm{AE}$ & $P$ value \\
\hline Training (s) & $65.10 \pm 39.03$ & $44.10 \pm 26.78$ & $79.00 \pm 22.45$ & $59.40 \pm 25.21$ & $66.88 \pm 26.19$ & $71.00 \pm 31.66$ & 0.1695 \\
\hline Test (24 h) & $62.10 \pm 29.18$ & $53.29 \pm 10.44$ & $86.78 \pm 19.85$ & $60.90 \pm 35.08$ & $67.25 \pm 28.67$ & $78.00 \pm 21.63$ & 0.1066 \\
\hline Test (7 d) & $41.00 \pm 18.93$ & $44.43 \pm 21.72$ & $62.22 \pm 19.33$ & $42.40 \pm 30.18$ & $57.75 \pm 22.16$ & $67.00 \pm 20.01$ & 0.0539 \\
\hline Test (14 d) & $39.60 \pm 12.82$ & $35.30 \pm 19.69$ & $52.70 \pm 21.33$ & $43.40 \pm 24.34$ & $55.13 \pm 19.53$ & $18.10 \pm 20.80$ & 0.0868 \\
\hline Test (21 d) & $44.10 \pm 12.35$ & $34.00 \pm 13.01$ & $45.90 \pm 8.54$ & $33.00 \pm 14.94$ & $48.33 \pm 18.25$ & $47.70 \pm 14.80$ & 0.0635 \\
\hline
\end{tabular}

TABLE 2: Maternal deprivation and physical exercise do not alter the anxiety of the animals. Data are expressed as the mean \pm SD of the time spent and the total number of entries in the open arms of the EPM. There was no significant difference between the groups on the different days (Kruskal-Wallis test; $n=8-10$ /group). $\mathrm{NMD}=$ nonmaternal-deprived rats; $\mathrm{MD}=$ maternal-deprived rats; $\mathrm{NMD}+\mathrm{FAE}=\mathrm{nonmaternal}$ deprived rats submitted to aerobic exercise familiarization; $\mathrm{MD}+\mathrm{FAE}=$ maternal-deprived rats submitted to aerobic exercise familiarization; $\mathrm{NMD}+\mathrm{AE}=$ nonmaternal-deprived rats submitted to aerobic exercise familiarization and to an aerobic exercise session; $\mathrm{MD}+\mathrm{AE}=$ maternal-deprived rats submitted to aerobic exercise familiarization and to an aerobic exercise session.

\begin{tabular}{|c|c|c|c|c|c|c|c|}
\hline & MD & NMD & $\mathrm{MD}+\mathrm{AE}$ & $\mathrm{NMD}+\mathrm{AE}$ & $\mathrm{MD}+\mathrm{FAE}$ & $\mathrm{NMD}+\mathrm{FAE}$ & $P$ value \\
\hline \multicolumn{8}{|l|}{$24 \mathrm{~h}$} \\
\hline Time in open arms & $138.7 \pm 19.1$ & $137.5 \pm 29.5$ & $145.0 \pm 26.6$ & $141.9 \pm 26.7$ & $146.0 \pm 31.1$ & $148.3 \pm 7.7$ & 0.75 \\
\hline Total of entries & $16.8 \pm 3.8$ & $15.2 \pm 5.1$ & $15.9 \pm 6.7$ & $17.2 \pm 6.4$ & $22.0 \pm 6.7$ & $22.80 \pm 5.4$ & 0.10 \\
\hline \multicolumn{8}{|l|}{7 days } \\
\hline Time in open arms & $143.7 \pm 18.0$ & $139.6 \pm 18.3$ & $136.7 \pm 22.3$ & $146.0 \pm 32.1$ & $151.9 \pm 19.1$ & $132.1 \pm 9.5$ & 0.21 \\
\hline Total of entries & $17.5 \pm 5.1$ & $20.0 \pm 5.4$ & $18.0 \pm 6.4$ & $15.8 \pm 5.0$ & $18.7 \pm 6.1$ & $20.1 \pm 4.1$ & 0.57 \\
\hline \multicolumn{8}{|l|}{14 days } \\
\hline Time in open arms & $133.7 \pm 16.1$ & $134.3 \pm 31.0$ & $142.2 \pm 34.9$ & $140.9 \pm 27.9$ & $155.0 \pm 20.0$ & $148.7 \pm 17.2$ & 0.35 \\
\hline Total of entries & $12.0 \pm 4.2$ & $12.0 \pm 3.3$ & $12.4 \pm 3.9$ & $13.2 \pm 4.2$ & $13.1 \pm 4.7$ & $13.5 \pm 4.7$ & 0.90 \\
\hline \multicolumn{8}{|l|}{21 days } \\
\hline Time in open arms & $145.0 \pm 30.1$ & $149.7 \pm 30.8$ & $150.3 \pm 41.5$ & $156.9 \pm 22.7$ & $148.7 \pm 17.7$ & $147.3 \pm 28.8$ & 0.95 \\
\hline Total of entries & $15.2 \pm 2.6$ & $14.0 \pm 2.6$ & $17.3 \pm 3.5$ & $17.3 \pm 2.5$ & $16.1 \pm 4.0$ & $15.5 \pm 5.2$ & 0.20 \\
\hline
\end{tabular}

Figure 3(b)). There are no differences between $\mathrm{MD}$ and $\mathrm{MD}+$ FAE groups $(P>0.05$, Figure $3(b))$ or $\mathrm{MD}+\mathrm{AE}$ group $(P>0.05$, Figure 3(b)).

\section{Discussion}

It is well established that the exposure to stress in the postnatal period can trigger numerous effects on neuroplasticity, cognitive function, and behavior in adulthood [44], since the postnatal period is critical for the central nervous system development [4]. In this sense, strategies that seek to minimize and/or reverse cognitive damages related to this type of stress are important. Here, we investigate the effects of an aerobic exercise (AE) shot on the consolidation and persistence of object recognition (OR) memory in a MD rat model. Our results showed that a single shot of exercise is able to modulate learning, promoting the acquisition of memory in MD rats, as well as the persistence of this memory up to 21 days. Additionally, the session of $\mathrm{AE}$ is able to improve the memory of NMD rats, since the memory persists for more time in these rats than in the ones that do not performed exercise. Still, the simple previous familiarization to the physical exercise apparatus to OR training promoted an improvement on learning.

Our results show that control animals (NMD) do not present memory persistence beyond 24 hours. It is importantly emphasized that it was expected, since this type of memory trace generally is susceptible to physiological decay [33]. On the other hand, MD rats are not able to learn, as previously demonstrated $[34,36,45]$. Aerobic exercise (AE) was able to revert $\mathrm{MD}$ memory deficits, and $\mathrm{MD}+\mathrm{AE}$ rats present better results than the NMD group in 7, 14, and 21 days because in control (NMD) rats, i.e., normal rats that were not submitted to any procedure despite the memory task, the OR memory trace normally decays along time. A single session of acute exercise, on the other hand, is aimed at modulating OR memory consolidation, and, as in NMD animals submitted to exercise, the MD animals that performed exercise had an improvement in consolidation with consequent improvement in the persistence of this memory. These 


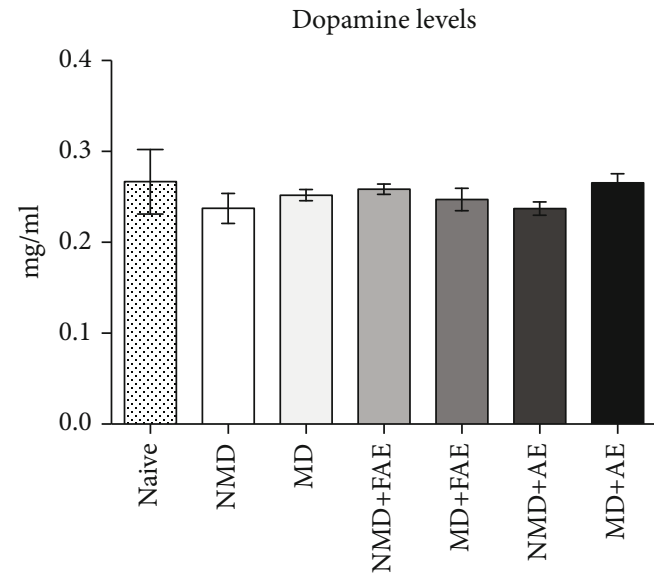

(a)

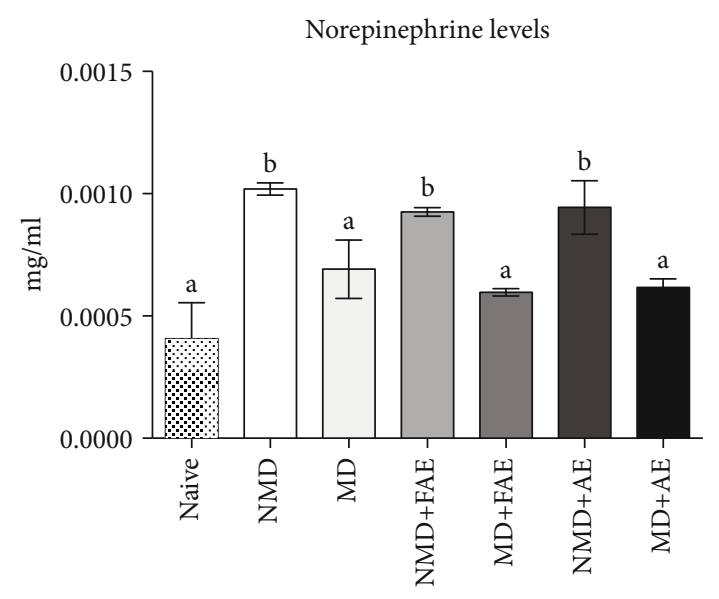

(b)

FIGURE 3: Hippocampal dopamine and norepinephrine levels on nonmaternal-deprived and maternal-deprived rats. Rats were trained in the OR task; some of them were submitted to an aerobic exercise shot, and after, hippocampus was removed. Bars depict HPLC dopamine (a) and norepinephrine (b) levels in hippocampal homogenates (mean \pm SD). All bars with letter " $A$ " are statistically equal and differ significantly to all bars with letter "B." $P \leq 0.05$ on ANOVA followed by Tukey's multiple comparison test; $n=3-5$ per group, analyzed in triplicate. $\mathrm{OR}=$ object recognition test; $\mathrm{NMD}=$ nonmaternal-deprived rats; $\mathrm{MD}=$ maternal-deprived rats; $\mathrm{NMD}+\mathrm{FAE}=$ nonmaternal-deprived rats submitted to aerobic exercise familiarization; $\mathrm{MD}+\mathrm{FAE}=$ maternal-deprived rats submitted to aerobic exercise familiarization; NMD+ $\mathrm{AE}=$ nonmaternal-deprived rats submitted to aerobic exercise familiarization and to an aerobic exercise session; $\mathrm{MD}+\mathrm{AE}=\mathrm{maternal}-$ deprived rats submitted to aerobic exercise familiarization and to an aerobic exercise session.

results are associated with the changes in brain activity after exercise [33], showing that improved memory consolidation may result in improved memory persistence [46]. This explains why $\mathrm{MD}+\mathrm{AE}$ animals show better memory than the NMD+FAE group, since these animals were not submitted to exercise after learning; so, memory consolidation was not modulated, and these animals show memory consolidation but did not show the same memory persistence (NMD+FAE). On the other hand, the MD+AE animals were the only that presented memory persistence until 21 days. Initially, we are expecting that the NMD+AE also presented this effect on memory, but it was not observed. We hypothesized that the brain changes induced by MD may make the brain of these animals more susceptible to changes after external influences, such as the practice of an exercise session [1]. One possible explanation to this result is that the neural changes induced by MD [1] may make the brain of these animals more susceptible to external influences along life or make them respond in a different way; so, factors as the practice of an exercise session could impact in a more expressive way the MD animals than control (NMD) ones.

Physical exercise is a noninvasive and easy alternative to influence the cognitive functions. Previous studies of our laboratory demonstrated that chronic aerobic exercise minimizes mnemonic deficits in animal models of neurodegenerative diseases $[24,36,47]$, which have been related to antioxidant and anti-inflammatory exercise effects [23, 27], besides the exercise influence on neuroplasticity [28]. However, acute aerobic exercise is still studied little as a neuroprotective strategy [48].

In a recent study, we demonstrated that normal rats submitted to one acute aerobic exercise session (30 minutes) after the OR learning present memory persistence [33]. The memory persistence induced by exercise was associated with the increase of NE levels in the hippocampus induced by exercise [33], since this neurotransmitter involvement on memory persistence is recognized [49]. Here, we confirmed that the aerobic exercise shot is able to improve memory, promoting its persistence, as previously reported [33], and, for the first time, we show that one exercise session can minimize the memory deficits related to maternal deprivation. $\mathrm{MD}$ rats that were not able to consolidate OR memory, when submitted to one session of exercise after OR learning, not only consolidate memory but also form a strong memory, which persists for 21 days, at least.

It is important to consider that our results show that the simple familiarization to the exercise apparatus had some effects on memory. Familiarization to exercise was able to improve memory persistence in NMD animals-at 7 and 14 days, but not at 21 , the same result of NMD+AE animals. Also, familiarization improved memory retention in MD animals ( $24 \mathrm{~h}$ test), which is not observed on MD animals not exposed to treadmill familiarization. This result could be attributed to a persistent effect of novelty (the physical exercise apparatus is a novelty for the rats), which has been shown to enhance short- and long-term memory by protein synthesis induction [45] or, what we consider more likely, to the familiarization to the treadmill, which could be considered as a short-term physical training and could induce some recognized physical exercise effects on the brain, as neuroplasticity, increasing brain blood supply, and others $[23,24$, $27,28,36,47]$. Initially, we included the familiarization group because the exercise group needed to be submitted to the familiarization to treadmill before the exercise session, anyway the animals would have difficulties on running, and they could be stressed, so we would like to guarantee that this procedure did not have effects per se. What we found is that 
aerobic exercise presented better effects than familiarization, especially on rats with memory deficit related to MD, but familiarization also promoted benefits to memory.

The physiological mechanisms involved in the effects of an acute aerobic exercise can be many; among them, it is known that exercise can promote increase of catecholamine levels as norepinephrine (NE) and dopamine (DA) [50], neurotransmitters involved on modulation of memory consolidation. In fact, we observed that all NMD groups showed increase of NE levels in comparison to the naïve group. However, we were not able to verify the same changes in hippocampal NE levels after OR training in MD rats or in the rats submitted to $\mathrm{AE}$ session. On the other hand, differences on DA levels were not observed between groups.

A previous study showed that MD causes a decrease in tyrosine hydroxylase enzyme in rodents [51], which is the first enzyme for dopaminergic metabolism, converting tyrosine into dopamine precursor DOPA [52]. Considering this finding, we could think that a decrease in NE levels in $\mathrm{MD}$ rats may be due to a gap in the enzyme; however, a decrease in tyrosine hydroxylase enzyme would also lead to a decrease in DA levels, which we could not demonstrate in this study. On the other hand, it is known that the main source of NE to the hippocampus is the locus coeruleus (LC) [21] a structure located in the brain stem, whose neurons are affected by MD. MD affects the dendritic morphology of the LC neurons [22], which may impair noradrenergic synapses in the hippocampus and could explain the impossibility to improve NE release in the hippocampus after OR training. This decrease of NE on MD rat hippocampus was not reversed by acute exercise, since one single physical exercise session is not able to promote immediate protein synthesis and neuroplasticity that would be required to revert this morphological alterations [53].

Moreover, the difference between DA and NE levels observed might be due to the incoordination between the control of noradrenergic systems, which is influenced by sympathoadrenal and central nervous systems, and dopaminergic, influenced exclusively by the central nervous system [54] previous works on MD models highlighted the impact of this intervention on the pituitary-adrenal axis, which could influence specially NE sources [55]. Corroborating to our work, a previous study with adult female rats showed that in the face of different MD protocols there were no significant differences in DA levels [56]; the authors highlighted that the duration of stress exposure and the time elapsed between stress exposure and euthanasia may influence these inconsistent findings. Another possible explanation for the discrepancy between this study and the others that find dopaminergic alterations on MD rats is the variation in age, as most studies use MD models in adolescent rats and we used young adults $[18,20,56]$.

Surprisingly, physical exercise did not promote NE alterations, as expected. These results also contradict previous results, although some of them use a single exercise session performed at high intensity without prior habituation, i.e., a protocol that differs a little from ours [31, 57]. This result suggests that increasing catecholamine levels could be related to the exercise intensity. Nonetheless, we can affirm that our results are specifically related to exercise effects, since, contrary to other studies, we did not use any type of aversive stimulus prior to or during the exercise session [58].

Despite the fact that we did not find significant differences in DA levels, we cannot assume that MD did not cause any disturbance on the dopaminergic system, as well as that the exercise did not influence this neurotransmitter release. It is important to highlight that the technique used can influence the measurements of DA and NE [59]. Although the HPLC technique used in our study had been previously used $[33,43]$, it is important to consider that the degradation of DA and NE can occur from 200 to 2000 milliseconds [52], which made their measurement difficult. So, measurements by in vivo microanalysis may provide more reliable results [60].

Finally, the improvement of cognitive deficits promoted by acute aerobic exercise may be associated with other factors that do not necessarily involve catecholamine modulation. Recent studies indicate that a single acute aerobic exercise session is effective to increase BDNF (brain-derived neurotrophic factor) mRNA, even in the absence of noradrenergic signaling [58]. BDNF is an important protein related to neural plasticity and closely related to long-lasting memory consolidation [61]. In addition, other studies suggest that epigenetic modulation through acute aerobic exercise [62] may induce histone acetylation, increasing HAT activity (histone acetyltransferases) in combination with the decrease of the activity of HDAC (histone deacetylases) in rat hippocampus, leading to neuroprotective effects [63].

In summary, acute aerobic exercise is an important procognitive stimulus that acts to attenuate cognitive deficits caused by MD. Despite the fact that we are not able to determine the exact modulatory mechanisms involved in the acute exercise effects, it is clearly an additional valuable measure to sustain the cognitive functions caused by early life stress.

\section{Conclusion}

MD promotes object recognition (OR) memory deficits. Acute physical exercise (AE) is an effective strategy to reverse recognition memory deficits related to $\mathrm{MD}$. After OR training, NMD rats present increase of NE, which is not observed in any MD groups. One session of physical exercise is not able to change DA or NE hippocampal levels, suggesting that the exercise effects on memory are related to other neural mechanisms.

\section{Data Availability}

Data will be made available on request.

\section{Additional Points}

Highlights. (1) Maternal deprivation causes persistent mnemonic deficits. (2) One aerobic exercise shot reduces mnemonic deficits induced by maternal deprivation. (3) One 
aerobic exercise shot contributes to memory persistence. (4) Maternal deprivation avoids the hippocampal norepinephrine increase induced by object recognition learning.

\section{Conflicts of Interest}

The authors declare that they have no conflicts of interest.

\section{Acknowledgments}

This study was financed in part by the Coordenação de Aperfeiçoamento de Pessoal de Nível Superior (CAPES/Brazil), finance code 001 and PROCAD program (grant number 88881.068493/2014-01), by the Universidade Federal do Pampa (UNIPAMPA/Brazil), by the Conselho Nacional de Pesquisa (CNPq/Brazil), and by the For Women in Science L'Oreal/UNESCO/ABC Program. PMS is supported by Fundação de Amparo à Pesquisa do Rio Grande do Sul (FAPERGS/Brazil), BHSN is supported by CAPES/Brazil, GSC is supported by $\mathrm{CNPq} / \mathrm{Brazil}$, GDR is supported by UNIPAMPA/Brazil, and PBMC is supported by $\mathrm{CNPq} / \mathrm{Bra}-$ zil and by UNIPAMPA/Brazil.

\section{References}

[1] X. Cao, S. Huang, J. Cao et al., "The timing of maternal separation affects morris water maze performance and long-term potentiation in male rats," Developmental Psychobiology, vol. 56, no. 5, pp. 1102-1109, 2014.

[2] R. Frau, S. Fanni, V. Serra et al., "Dysfunctional mesocortical dopamine circuit at pre-adolescence is associated to aggressive behavior in MAO-A hypomorphic mice exposed to early life stress," Neuropharmacology, vol. 159, p. 107517, 2019.

[3] A. Knafo and S. R. Jaffee, "Gene-environment correlation in developmental psychopathology," Development and Psychopathology, vol. 25, no. 1, pp. 1-6, 2013.

[4] D. M. de Campo, J. L. Cameron, J. M. Miano, D. A. Lewis, K. Mirnics, and J. L. Fudge, "Maternal deprivation alters expression of neural maturation gene tbr1 in the amygdala paralaminar nucleus in infant female macaques," Developmental Psychobiology, vol. 59, no. 2, pp. 235-249, 2017.

[5] E. M. Marco, R. Llorente, M. López-Gallardo et al., "The maternal deprivation animal model revisited," Neuroscience \& Biobehavioral Reviews, vol. 51, pp. 151-163, 2015.

[6] R. J. Mclaughlin and G. GOBBI, "Cannabinoids and emotionality: a neuroanatomical perspective," Neuroscience, vol. 204, pp. 134-144, 2012.

[7] J. Bruce, A. R. Tarullo, and M. R. Gunnar, "Disinhibited social behavior among internationally adopted children," Development and Psychopathology, vol. 21, no. 1, pp. 157-171, 2009.

[8] Schizophrenia Working Group of the Psychiatric Genomics Consortium, "Biological insights from 108 schizophreniaassociated genetic loci," Nature, vol. 511, no. 7510, pp. 421427, 2014.

[9] E. D. Abercrombie, K. A. Keefe, D. S. DiFrischia, and M. J. Zigmond, "Differential effect of stress on in vivo dopamine release in striatum, nucleus accumbens, and medial frontal cortex," Journal of Neurochemistry, vol. 52, no. 5, pp. 1655-1658, 1989.

[10] J. D. Berke, "What does dopamine mean?," Nature Neuroscience, vol. 21, no. 6, pp. 787-793, 2018.
[11] Z. Kasanova, D. Hernaus, T. Vaessen et al., "Early-life stress affects stress-related prefrontal dopamine activity in healthy adults, but not in individuals with psychotic disorder," PLoS One, vol. 11, no. 3, p. e0150746, 2016.

[12] A. S. Loria, M. W. Brands, D. M. Pollock, and J. S. Pollock, "Early life stress sensitizes the renal and systemic sympathetic system in rats," American Journal of Physiology-Renal Physiology, vol. 305, no. 3, pp. F390-F395, 2013.

[13] L. Pani, A. Porcella, and G. L. Gessa, "The role of stress in the pathophysiology of the dopaminergic system," Molecular Psychiatry, vol. 5, no. 1, pp. 14-21, 2000.

[14] A. Bjorklund and S. B. Dunnett, "Dopamine neuron systems in the brain: an update," Trends in Neurosciences, vol. 30, no. 5, pp. 194-202, 2007.

[15] M. Fitzgerald, "The development of nociceptive circuits," Nature Reviews Neuroscience, vol. 6, no. 7, pp. 507-520, 2005.

[16] M. K. Fitzgerald, J. M. Otis, and D. Mueller, "Dissociation of $\beta 1$ - and $\beta 2$-adrenergic receptor subtypes in the retrieval of cocaine-associated memory," Behavioural Brain Research, vol. 296, pp. 94-99, 2016.

[17] W. Schultz, "Multiple dopamine functions at different time courses," Annual Review of Neuroscience, vol. 30, no. 1, pp. 259-288, 2007.

[18] I. Majcher-Maślanka, A. Solarz, K. Wędzony, and A. Chocyk, "The effects of early-life stress on dopamine system function in adolescent female rats," International Journal of Developmental Neuroscience, vol. 57, pp. 24-33, 2017.

[19] H. Ilchmann-Diounou, M. Olier, C. Lencina et al., "Early life stress induces type 2 diabetes-like features in ageing mice," Brain, Behavior, and Immunity, vol. 80, pp. 452-463, 2019.

[20] J. B. Dwyer and F. M. Leslie, "Adolescent maturation of dopamine D1 and D2 receptor function and interactions in rodents," PLoS One, vol. 11, no. 1, p. e0146966, 2016.

[21] T. L. Sterley, F. M. Howells, and V. A. Russell, "Maternal separation increases $\mathrm{GABA}_{\mathrm{A}}$ receptor-mediated modulation of norepinephrine release in the hippocampus of a rat model of ADHD, the spontaneously hypertensive rat," Brain Research, vol. 1497, pp. 23-31, 2013.

[22] J. D. Swinny, E. O'Farrell, B. C. Bingham, D. A. Piel, R. J. Valentino, and S. G. Beck, "Neonatal rearing conditions distinctly shape locus coeruleus neuronal activity, dendritic arborization, and sensitivity to corticotrophin-releasing factor," International Journal of Neuropsychopharmacology, vol. 13, no. 04, pp. 515-525, 2010.

[23] W. Chen, J. Li, J. Liu, D. Wang, and L. Hou, “Aerobic exercise improves food reward systems in obese rats via insulin signaling regulation of dopamine levels in the nucleus accumbens," ACS Chemical Neuroscience, vol. 10, no. 6, pp. 2801-2808, 2019.

[24] P. M. Sosa, H. L. Schimidt, C. Altermann et al., "Physical exercise prevents motor disorders and striatal oxidative imbalance after cerebral ischemia-reperfusion," Brazilian Journal of Medical and Biological Research, vol. 48, no. 9, pp. 798-804, 2015.

[25] M. Asztalos, I. De Bourdeaudhuij, and G. Cardon, "The relationship between physical activity and mental health varies across activity intensity levels and dimensions of mental health among women and men," Public Health Nutrition, vol. 13, no. 8, pp. 1207-1214, 2010.

[26] C. H. Chu, A. F. Kramer, T. F. Song, C. H. Wu, T. M. Hung, and Y. K. Chang, "Acute exercise and neurocognitive development in preadolescents and young adults: an ERP study," 
Neural Plasticity, vol. 2017, Article ID 2631909, 13 pages, 2017.

[27] L. M. S. Cordeiro, P. C. R. Rabelo, M. M. Moraes et al., "Physical exercise-induced fatigue: the role of serotonergic and dopaminergic systems," Brazilian Journal of Medical and Biological Research, vol. 50, no. 12, 2017.

[28] A. Dinoff, N. Herrmann, W. Swardfager, and K. L. Lanctôt, "The effect of acute exercise on blood concentrations of brain-derived neurotrophic factor in healthy adults: a metaanalysis," European Journal of Neuroscience, vol. 46, no. 1, pp. 1635-1646, 2017.

[29] T. McMorris, K. Collard, J. Corbett, M. Dicks, and J. P. Swain, "A test of the catecholamines hypothesis for an acute exercisecognition interaction," Pharmacology Biochemistry and Behavior, vol. 89, no. 1, pp. 106-115, 2008 .

[30] T. McMorris and J. Graydon, "The effect of exercise on cognitive performance in soccer-specific tests," Journal of Sports Sciences, vol. 15, no. 5, pp. 459-468, 1997.

[31] R. Pagliari and L. Peyrin, "Norepinephrine release in the rat frontal cortex under treadmill exercise: a study with microdialysis," Journal of Applied Physiology, vol. 78, no. 6, pp. 21212130, 1995.

[32] D.'e. Sutoo and K. Akiyama, "Regulation of brain function by exercise," Neurobiology of Disease, vol. 13, no. 1, pp. 1-14, 2003.

[33] L. da Silva de Vargas, B.-H. S. d. Neves, R. Roehrs, I. Izquierdo, and P. Mello-Carpes, "One-single physical exercise session after object recognition learning promotes memory persistence through hippocampal noradrenergic mechanisms," Behavioural Brain Research, vol. 329, pp. 120-126, 2017.

[34] F. Benetti, P. B. Mello, J. S. Bonini, S. Monteiro, M. Cammarota, and I. Izquierdo, "Early postnatal maternal deprivation in rats induces memory deficits in adult life that can be reversed by donepezil and galantamine," International Journal of Developmental Neuroscience, vol. 27, no. 1, pp. 5964, 2009.

[35] P. B. Mello, F. Benetti, M. Cammarota, and I. Izquierdo, "Physical exercise can reverse the deficit in fear memory induced by maternal deprivation," Neurobiology of Learning and Memory, vol. 92, no. 3, pp. 364-369, 2009.

[36] B. H. Neves, J. Menezes, M. A. Souza, and P. B. Mello-Carpes, "Physical exercise prevents short and long-term deficits on aversive and recognition memory and attenuates brain oxidative damage induced by maternal deprivation," Physiology \& Behavior, vol. 152, no. Part A, pp. 99-105, 2015.

[37] R. M. Arida, F. A. Scorza, S. G. da Silva, R. M. Cysneiros, and E. A. Cavalheiro, "Exercise paradigms to study brain injury recovery in rodents," American Journal of Physical Medicine \& Rehabilitation, vol. 90, no. 6, pp. 452-465, 2011.

[38] G. A. Brooks and T. P. White, "Determination of metabolic and heart rate responses of rats to treadmill exercise," Journal of Applied Physiology, vol. 45, no. 6, pp. 1009-1015, 1978.

[39] F. Cechetti, P. V. Worm, V. R. Elsner et al., "Forced treadmill exercise prevents oxidative stress and memory deficits following chronic cerebral hypoperfusion in the rat," Neurobiology of Learning and Memory, vol. 97, no. 1, pp. 90-96, 2012.

[40] A. Ennaceur and J. Delacour, "A new one-trial test for neurobiological studies of memory in rats. 1: behavioral data," Behavioural Brain Research, vol. 31, no. 1, pp. 47-59, 1988.
[41] M. H. Malek, M. Hüttemann, I. Lee, and J. W. Coburn, "Similar skeletal muscle angiogenic and mitochondrial signalling following 8 weeks of endurance exercise in mice: discontinuousversuscontinuous training," Experimental Physiology, vol. 98, no. 3, pp. 807-818, 2013.

[42] S. Pellow, P. Chopin, S. E. File, and M. Briley, "Validation of open : closed arm entries in an elevated plus-maze as a measure of anxiety in the rat," Journal of Neuroscience Methods, vol. 14, no. 3, pp. 149-167, 1985.

[43] J. Menezes, N. Alves, S. Borges et al., "Facilitation of fear extinction by novelty depends on dopamine acting on D1subtype dopamine receptors in hippocampus," Proceedings of the National Academy of Sciences, vol. 112, no. 13, pp. E1652-E1658, 2015.

[44] M. O. Albuquerque Filho, B. S. de Freitas, R. C. L. Garcia, P. C. .F. Crivelaro, N. Schröder, and M. N. M. de Lima, "Dual influences of early-life maternal deprivation on histone deacetylase activity and recognition memory in rats," Neuroscience, vol. 344, pp. 360-370, 2017.

[45] J. Menezes, B. H. Neves, M. Souza, and P. B. Mello-Carpes, "Green tea protects against memory deficits related to maternal deprivation," Physiology \& Behavior, vol. 182, pp. 121-127, 2017.

[46] J. L. McGaugh, "Memory-a century of Consolidation," Science, vol. 287, no. 5451, pp. 248-251, 2000.

[47] L. Rossi Dare, A. Garcia, N. Alves, D. Ventura Dias, M. A. de Souza, and P. B. Mello-Carpes, "Physical and cognitive training are able to prevent recognition memory deficits related to amyloid beta neurotoxicity," Behavioural Brain Research, vol. 365, pp. 190-197, 2019.

[48] F. de Jesus Furtado Almeida, B. B. Gambassi, P. A. Schwingel et al., "Possible benefits of different physical exercise programs after coronary artery bypass graft surgery: a minireview of selected randomized controlled trials," Sport Sciences for Health, vol. 13, no. 3, pp. 477-483, 2017.

[49] P. B. Mello-Carpes, L. da Silva de Vargas, M. C. Gayer, R. Roehrs, and I. Izquierdo, "Hippocampal noradrenergic activation is necessary for object recognition memory consolidation and can promote BDNF increase and memory persistence," Neurobiology of Learning and Memory, vol. 127, pp. 84-92, 2016.

[50] B. Winter, C. Breitenstein, F. C. Mooren et al., "High impact running improves learning," Neurobiology of Learning and Memory, vol. 87, no. 4, pp. 597-609, 2007.

[51] I. S. Pienaar, L. A. Kellaway, V. A. Russell et al., "Maternal separation exaggerates the toxic effects of 6-hydroxydopamine in rats: implications for neurodegenerative disorders," Stress, vol. 11, no. 6, pp. 448-456, 2008.

[52] H. Juárez Olguín, D. Calderón Guzmán, E. Hernández García, and G. Barragán Mejía, "The role of dopamine and its dysfunction as a consequence of oxidative stress," Oxidative Medicine and Cellular Longevity, vol. 2016, Article ID 9730467, 13 pages, 2016.

[53] K. Knaepen, M. Goekint, E. M. Heyman, and R. Meeusen, "Neuroplasticity - exercise-induced response of peripheral brain-derived neurotrophic factor," Sports Medicine, vol. 40, no. 9, pp. 765-801, 2010.

[54] K. Gogberashvili, "The comparative effect of continuous and intermittent maternal separation on catecholamines blood concentrations among rat pups," Georgian Medical News, no. 142 , pp. 70-72, 2007. 
[55] R. H. A. van der Doelen, W. Deschamps, C. D'Annibale et al., "Early life adversity and serotonin transporter gene variation interact at the level of the adrenal gland to affect the adult hypothalamo-pituitary- adrenal axis," Translational Psychiatry, vol. 4, no. 7, p. e409, 2014.

[56] L. Arborelius and M. B. Eklund, "Both long and brief maternal separation produces persistent changes in tissue levels of brain monoamines in middle-aged female rats," Neuroscience, vol. 145, no. 2, pp. 738-750, 2007.

[57] R. Pagliari and L. Peyrin, "Physical conditioning in rats influences the central and peripheral catecholamine responses to sustained exercise," European Journal of Applied Physiology and Occupational Physiology, vol. 71, no. 1, pp. 41-52, 1995.

[58] A. C. Venezia, E. Quinlan, and S. M. Roth, "A single bout of exercise increases hippocampalBdnf: influence of chronic exercise and noradrenaline," Genes, Brain and Behavior, vol. 16, no. 8, pp. 800-811, 2017.

[59] J. J. Kirkland, "Ultrafast reversed-phase high-performance liquid chromatographic separations: an overview," Journal of Chromatographic Science, vol. 38, no. 12, pp. 535-544, 2000.

[60] E. Dremencov, L. Lacinova, G. Flik, J. H. A, T. I. F, and B. H. C, "Purinergic regulation of brain catecholamine neurotransmission: In vivo electrophysiology and microdialysis study in rats," General Physiology and Biophysics, vol. 36, no. 04, pp. 431-441, 2017.

[61] P. Bekinschtein, M. Cammarota, and J. H. Medina, "BDNF and memory processing," Neuropharmacology, vol. 76, no. Part C, pp. 677-683, 2014.

[62] V. R. Elsner, G. A. Lovatel, K. Bertoldi et al., "Effect of different exercise protocols on histone acetyltransferases and histone deacetylases activities in rat hippocampus," Neuroscience, vol. 192, pp. 580-587, 2011.

[63] V. R. Elsner, C. Basso, K. Bertoldi, L. C. F. de Meireles, L. R. Cechinel, and I. R. Siqueira, "Differential effect of treadmill exercise on histone deacetylase activity in rat striatum at different stages of development," The Journal of Physiological Sciences, vol. 67, no. 3, pp. 387-394, 2017. 


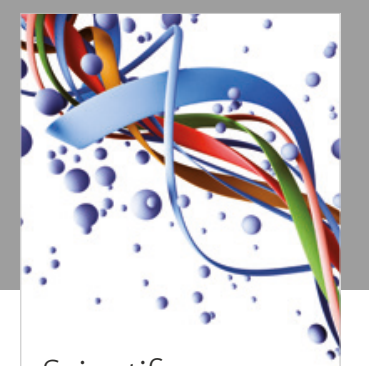

Scientifica
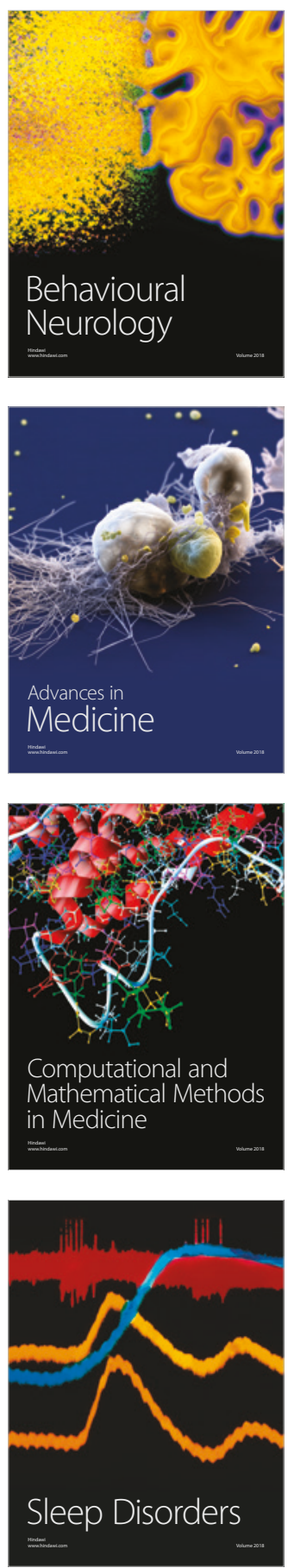

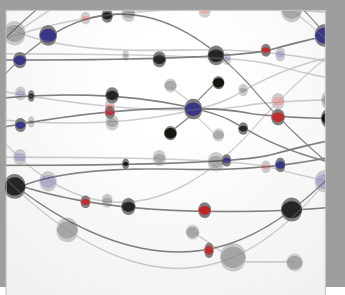

The Scientific World Journal

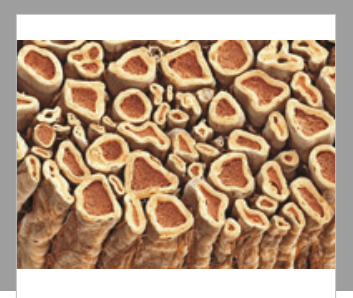

Case Reports in

Neurological Medicine

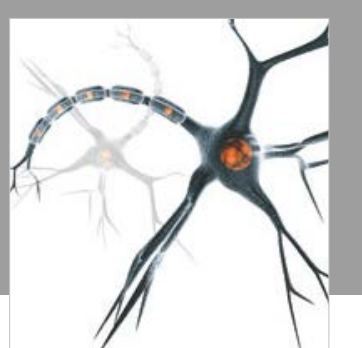

Neural Plasticity

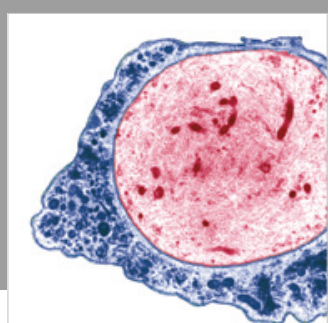

Multiple Sclerosis

International

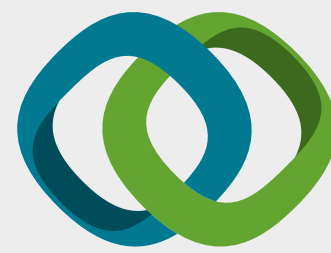

Hindawi

Submit your manuscripts at

www.hindawi.com
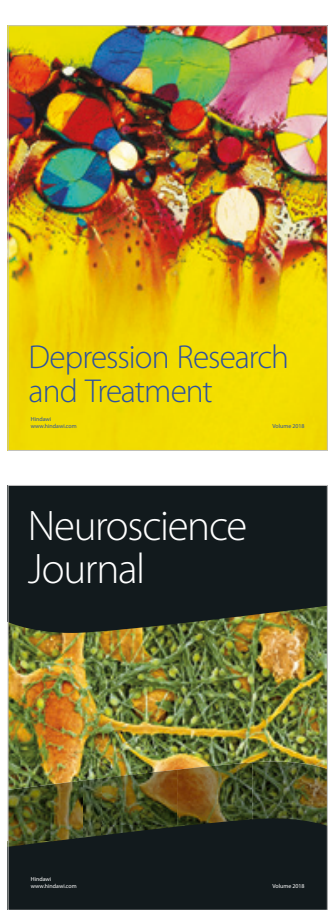

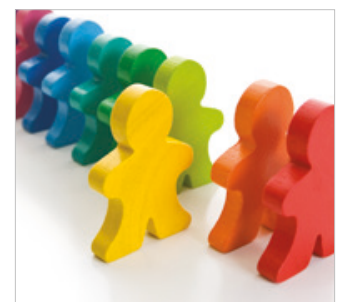

Autism

Research and Treatment
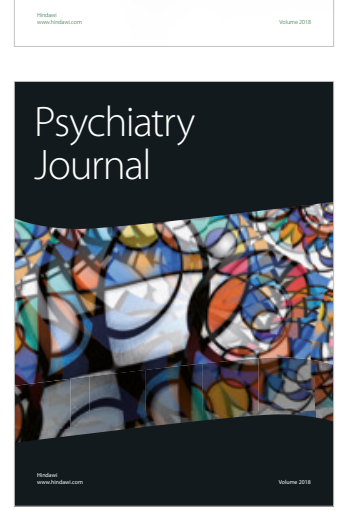
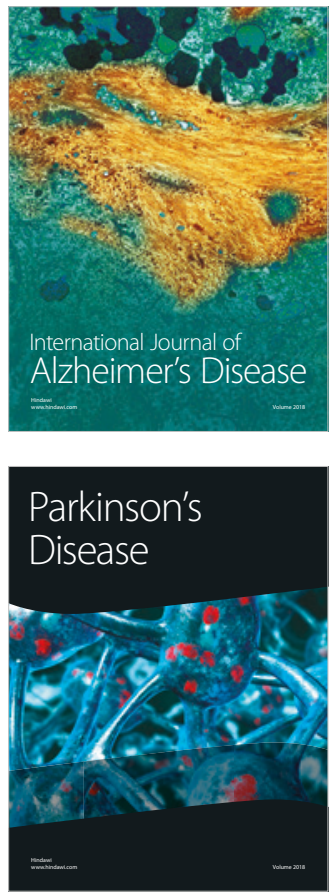
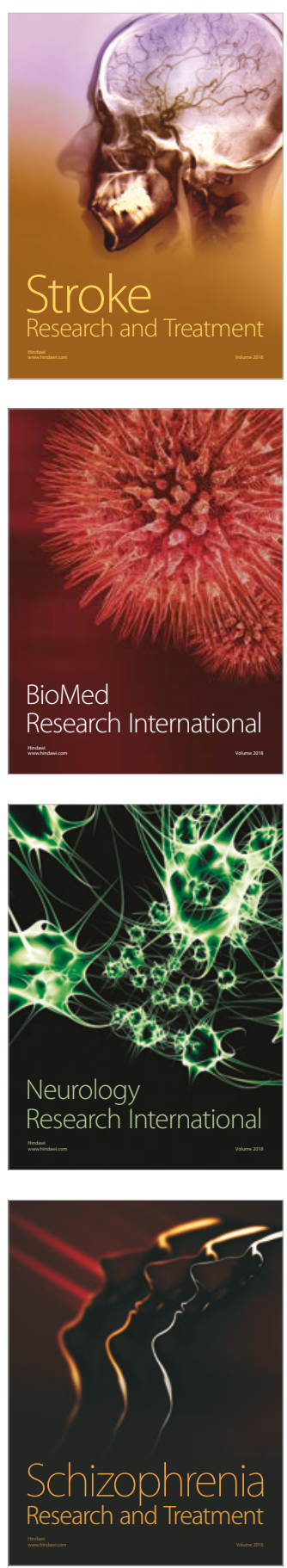